\title{
"A Single Blinded Comparative Study of Diclofenac Gel And Evening Primrose oil In Management of Women Presenting With Mastalgia"
}

\author{
Dr. Rajeev Thilak ${ }^{1}$, Dr. Ameesh Isath ${ }^{2}$, Dr.S. Thirunavukkarasu ${ }^{2}$, \\ Dr. Dhayanathi Dhayalan ${ }^{3}$ \\ ${ }^{I}$ (Institute of general surgery/ Madras Medical College, India) \\ ${ }^{2}($ (Institute of general surgery/ Madras Medical College, India) \\ ${ }^{3}$ (Institute of general surgery/ Madras Medical College, India) \\ ${ }^{4}$ (Institute of general surgery/Madras Medical College, India)
}

\begin{abstract}
Breast pain or mastalgia is the most common clinical problems with which most of the women approach to our surgical outpatient department. Several treatments have been proposed in management of mastalgia but yet recently diclofenac gel has been proven to be effective in treatment of mastalgia. This study is to compare the effectiveness of diclofenac gel and evening primrose oil in management of mastalgia. Between march 2016 and September 2016, 80 patients with mastalgia who got consultation as outpatient or inpatient in Institute of General Surgery, Rajiv Gandhi Government General Hospital, Chennai were subjected to evaluation and management as per treatment guidelines derived from recent systematic review. Data was collected and analyzed.

Majority of the patients presented with fear of cancer rather than debilitating pain which would hinder with their activities of daily life. Diclofenac gel was found to reduce pain more effectively than evening primrose oil in patient presenting with mastalgia after monitoring for a period of 6 months. Patient education and availability of quality radiological and pathological services are crucial in the management of patients with mastalgia in alleviating the fear of cancer among these women. Reassurance and appropriate medical and surgical treatment according to the cause of breast pain can reduce the morbidity and improve the outcome of the disease. In the end it was concluded that Diclofenac gel was proven to be more effective than evening primrose oil in management of mastalgia. There was significant reduction in pain when topical diclfenac gel was used when compared to Evening primrose oil. The pain score was monitored by Visual Analogue Score
\end{abstract}

Keyword: mastalgia, breast pain, analgesics, breast support, cancer fear, Visual Analogue Scale

\section{Introduction}

Breast pain is the most common breast symptom causing women to consult physicians and surgeons. It is rarely a sign of cancer. However, concern about cancer is the primary reason for most women to seek medical evaluation and treatment for this syndrome (Eberl et al. 2008). Breast pain is classified as cyclic mastalgia, noncyclical mastalgia, and chest wall pain. Cyclic mastalgia is a breast pain occurring in a predictable pattern with the menstrual cycle, often a few days before menstruation. Noncyclical mastalgia is described as a constant or intermittent pain with irregular exacerbations and no relationship to menstruation. Although women with chest wall pain may complain of breast pain, the actual source of the pain is not the breast tissue but rather the costochondral joint underlying it.

Mastalgia is cyclic in two-thirds of the patients (Mansel 1994). Cyclic pain is often bilateral, usually most severe in the upper outer quadrants of the breast, and may be referred to the upper arm and axilla. According to severity breast pain can be classified as mild, moderate or severe. Mild premenstrual discomfort lasting for one to four days can be considered normal. Research criteria for the diagnosis of severe cyclic mastalgia include pain severity greater than $4 \mathrm{~cm}$ measured with a $10 \mathrm{~cm}$ VAS and pain duration of at least 7 days per month (Ader and Browne 1997a). Approximately 8-10\% of premenopausal women experience moderate to severe breast pain monthly during the premenstrual period (Ader et al. 1999). Premenstrual mastalgia has been reported to interfere remarkably with women's lives, relationships, work and sleep (Ader and Shriver 1997b).

The evaluation of mastalgia begins with a thorough history and a careful physical examination. Special attention should be given to the type of pain, its location and its relation to the menstrual cycle. Women with noncyclical mastalgia will benefit from a physical examination to determine whether the pain comes from the breast or chest wall. Although cyclic mastalgia is a well-known somatic component of the premenstrual 
syndrome (PMS), more than $80 \%$ of women with premenstrual mastalgia do not meet the criteria for PMS (Ader et al. 1999).

A higher use of mammography among young women with mastalgia compared with age-matched women without mastalgia has been observed (Ader and Browne 1997a). The particular value of breast imaging in breast pain is reassurance for both the patient and physician. According to severity, breast pain can be classified as mild, moderate or severe. The reported prevalence among premenopausal women ranges from $41 \%$ to $79 \%$ [1]. According to Ader and South-Paul (2001) reported that premenstrual discomfort was present among $70 \%$ of the premenstrual women. In a large clinic-based trial of 1,171 women attending a gynaecologic clinic for any reason, $69 \%$ suffered from regular discomfort, which was judged as severe by $11 \%$ of women, and 36 $\%$ had consulted a doctor for the breast pain (Ader and Browne 1997a) [2]. Moderate to severe breast pain during the premenstrual period is experienced monthly by approximately 8-10\% of premenopausal women (Ader et al. 1999) [2]. In another clinic-based trial of 231 women, cyclic mastalgia was reported to interfere with sexual activity by $48 \%$, with physical activity by $36 \%$, with work, school or social activities by $6 \%$ to $13 \%$, and with sleep by $10 \%$ of the women, respectively. Moreover, $79 \%$ of the participants had regularly experienced cyclic breast pain and nearly half of the study population had asked some healthcare provider about their symptoms (Ader and Shriver 1997b). The average age of the patients is 34 years who present with the cyclic type of breast pain. Noncyclical mastalgia are often found among later age usually around fourth or fifth decade of life at presentation (BeLieu 1994, Mansel 1994) [3]. An Australian study of 170 mastalgia patients reported that the average age of the women was 42 years and that the breast pain was cyclic in $59 \%$ of the cases [4]. The patients of this trial attended a specific mastalgia clinic over a three-year period (Wetzig 1994). Current moderate to severe premenstrual mastalgia lasting for five days or more monthly was reported by one third of the study population. An onset of cyclic pain before the age of 20 years was followed by a prolonged course. An improvement in the pain was spontaneous in $22 \%$ of the cases and resulted from a hormonally related event like menopause, pregnancy, or the use of oral contraceptives. In a long-term follow up study of 212 women, Davies et al. (1998) found that the patients with cyclic mastalgia tended to develop their pain earlier than noncyclical mastalgia patients, in accordance with BeLieu (1994) and Mansel (1994) [4].

Although breast pain was long-lasting in both groups (median 12 years), it was longer in patients with cyclic mastalgia, who also developed breast pain earlier [4]. The rate of spontaneous resolution (40 \%) was found to be higher in non cyclical mastalgia than in the cyclic group (14\%). In most patients the resolution of cyclic mastalgia was associated with the menopause. In a prospective study of 72 patients, 35 patients were suffering from the noncyclical mastalgia and 37 from the musculoskeletal pain, the latter including Tietze's syndrome and other causes of chest wall pain [5]. The mean age of the participants at presentation was greater in the musculoskeletal group (39.3 vs. 33.9 years) with a shorter mean duration of pain (14.7 vs. 35.4 months) as compared with the noncyclical mastalgia group. The most common breast symptom is breast pain causing women to consult primary care physicians and surgeons. A retrospective cohort study with 2,400 pre- and postmenopausal women found that women younger than 50 years of age presented with breast symptoms to their primary care physician twice as often as older women did [6]. Additionally, subjects with a family history of breast cancer $(22 \%)$ were more likely to present with breast complaints compared with those without a family history (14\%) (Barton et al. 1999a). On the other hand, Nichols et al. (1980) reported that approximately one third of the breast symptom visits to general practices were made by women younger than 35 years of age. In the primary care setting, breast symptoms were reported in about $3 \%$ of all visits by female patients [7].

The breast pain $(48 \%)$ and a breast mass $(29 \%)$ were the most common breast-related complaints. Complaints of breast symptoms were most common among women aged 25 to 44 years (48 of 1,000) and among women aged 65 years and older (33 per 1,000), respectively [8]. This 17-year data on the prevalence of breast symptoms is based on a population of 84,285 female patients (Eberl et al. 2008). In a prospective study with 30 women, it was found that most women with mastalgia had often also other menstrual complaints [8]. However, $12 \%$ of the patients with a severe cyclic mastalgia had relatively minimal associated symptoms (Tavaf-Motamen et al. 1998). The following measures can be applied to both noncyclical and cyclical mastalgia. Daily Breast Pain Charting: Patients are explained about hormonal changes with menstruation and its relation with mastalgia and requested to record their daily pain experience on a chart (Fig. 1). The days with menses are marked with letter "P".

The severity of pain is also noted on a visual analogue scale (VAS, also called visual linear analogueue). VAS is usually a horizontal line, $10 \mathrm{~cm}$ in length, anchored by word descriptors at each end. The patient marks on the line below, the point that they feel represents their perception of pain. The VAS score is determined by measuring in millimeter from the left hand end of the line to the point that the patient marks. On this VAS, the 0 indicates no pain and 10 indicate very severe excruciating pain in the breast [9]. Any pain of $\geq 3$ on a VAS of 0 to 10 to be significantly severe and usually require therapy.

\section{Reassurance:}


Reassurance can be given to those women with no abnormality detected on triple assessment that the possibility of breast cacinoma is less than $5 \%$ as most of the women get relieved with such a message.

Physical measures:

It is estimated that up to $70 \%$ of women wear improperly fitted bras. Thus, it is important to ensure that the patient is fitted with sufficiently sup- portive and well-fitting brassiere. It is very useful those women with large breast. a randomized trial of 200 women with mastalgia, 100 women received treatment with danazol and the other 100 were asked to wear sports brassieres for 12 weeks. The danazol group had $58 \%$ relief of symptoms (with drug side effects in $42 \%$ ), while in the brassiere group, $85 \%$ had relief of symptoms with improved lifestyle [9]. Sports garments reduces the over stretching of the cooper's ligament thereby relieving the pain. Relaxation techniques: Four weeks of relaxation therapy was reported to provide relief in $60 \%$ of patients [9].

Vitamins: There is no convincing evidence to support the use of vitamins in treatment of mastalgia; however, vitamin B1, B6, and E are all used by clinicians. These have no role and should not be misused [9]. A recent meta-analysis described that long-term use of vitamin $\mathrm{E}$ may increase risk of hemorrhagic stroke $(\mathrm{RR}=$ $1.22 ; 95 \%$ confidence interval 1 to $1.48 ; \mathrm{p}=0.045$ ) [10].

Evening primrose oil: Although evening primrose oil has been used in past with some success in a few trials $[9,11]$, meta-analysis revealed that the benefit in pain reduction is similar to that achieved by placebo [9]. But some centers still prefer to use evening primrose oil for breast pain

\section{Dietary habits:}

Based on epidemiological studies, regular, moderate physical exercise has a positive effect on menstrual cycle-related symptoms and mood states[12]. Dietary changes often recommended as a treatment of premenstrual symptoms include reduction of salt, sugar, alcohol, and caffeine intake. Consumption of an evening meal rich in carbohydrate and poor in protein during the late luteal phase seems to be effective in PMSrelated mood disorders [12]. Avoidance of caffeine has been shown to have no benefit [1]. A lack of essential fatty acids, or linoleic and gamma linoleic acid, has been implicated in the aetiology of perimenstrual symptoms. Linoleic acids, found in fish oil, inhibit arachidonic acid formation at the beginning of the prostaglandins cascade, and have been found to relieve pain symptoms. Although gamma linoleic acid is a precursor for prostaglandins, some studies have shown that increasing gamma linoleic acid intake reduces premenstrual breast tenderness and fluid retention. Treatment with primrose oil has found to produce a significant increase in the proportion of plasma essential fatty acid esters. Clinical response to primrose oil in mastalgia was better in patients with lower pretreatment levels of the esters of the unsaturated fatty acids [12].

\section{Topical NSAIDs \Diclofenac gel:}

Upto $80 \%$ of women benefit with use of Non Steroidal Anti-medications but their benefits are usually underestimated. Diclofenac gel applied as local massage to painful areas of breast has been found to be more effective than placebo gel and ibuprofen gel in randomized trials [13]. Colak studied the effects of topical diclofenac gel on cyclic and noncyclical mastalgia in 108 patients: 60 with cyclic (group I) and 48 with noncyclical (group II). Patients within each group were randomized to diclofenac gel or placebo cream, three times daily for 6 months [13]. The pain score decreased significantly in diclofenac gel group compared to placebo. The benefits were seen in cyclical and non- cyclical breast pain. No side effect was reported in any group [13].

\section{Methods}

In this clinical study on women presenting with mastalgia, datas from the patient were collected after obtaining proper written informed consent from the patients or their guardians and analysed in comparison to the literature. The main epidemiological parameters which were included in this study were age, body mass index, dietary habits, complaint of lumpiness of the breast, type of mastalgia whether cyclical or non-cyclical, menstrual habits and previous history of benign breast diseases. These patients undergo thorough breast examination and general examination and systematic examination and other findings noted. Patients were classified by their age groups and body mass index calculated by obtaining weight in kilogram and height in meter. Dietary habits were also taken into account and mainly divided as vegetarians and non vegeterians. Complaint of lumpiness of one or both breasts was obtained and palpation of the breasts was carried out accordingly. Type of mastalgia was elicited by maintaining breast pain chart and relation to the mensus was noted as cyclical when it occurs 1-2 weeks prior to mensus and non-cyclical otherwise. Previous history of similar complaints in the past including previous medical or surgical treatment for the same was also noted. Brestfeeding habits were also noted among the married women. 
Clinical breast examination findings were noted as absence of significant abnormality or lump or nodularity. When a lump in the breast was present, it was investigated by international protocol of triple assessment by clinical, radiological and pathological means. All these patients who mostly present with fear of cancer are comforted with an ultrasound scan of the breast and axilla. These findings are noted as absence of significant abnormality or fibroadenoma [FA], fibrocystic changes [FC] or abscess. Patient with nodularity or lump in clinical examination or by radiology were subjected to FNAC. Patients with mastalgia were then given treatment either with topical diclofenac gel or evening primrose oil. Response is noted in terms of visual analogue scores of breast pain. The data thus obtained in 100 patients as prospective observational study over a period of 6 months study duration are analysed for patterns and relations. VAS is usually noted at start and at the end of follow up. Effectiveness of diclofenac gel and evening primrose oil was evaluated and compared.

\subsection{Age distribution and mastalgia}

\section{Results}

\begin{tabular}{|l|l|l|}
\hline \multicolumn{1}{|c|}{ Age } & $(\mathrm{n}=100)$ & Percentage \\
\hline & & \\
\hline $15-20$ & 37 & $37 \%$ \\
\hline $21-25$ & 34 & $34 \%$ \\
\hline $26-30$ & 15 & $15 \%$ \\
\hline $30-35$ & 7 & $7 \%$ \\
\hline $36-40$ & 5 & $5 \%$ \\
\hline $41-45$ & 2 & $2 \%$ \\
\hline
\end{tabular}

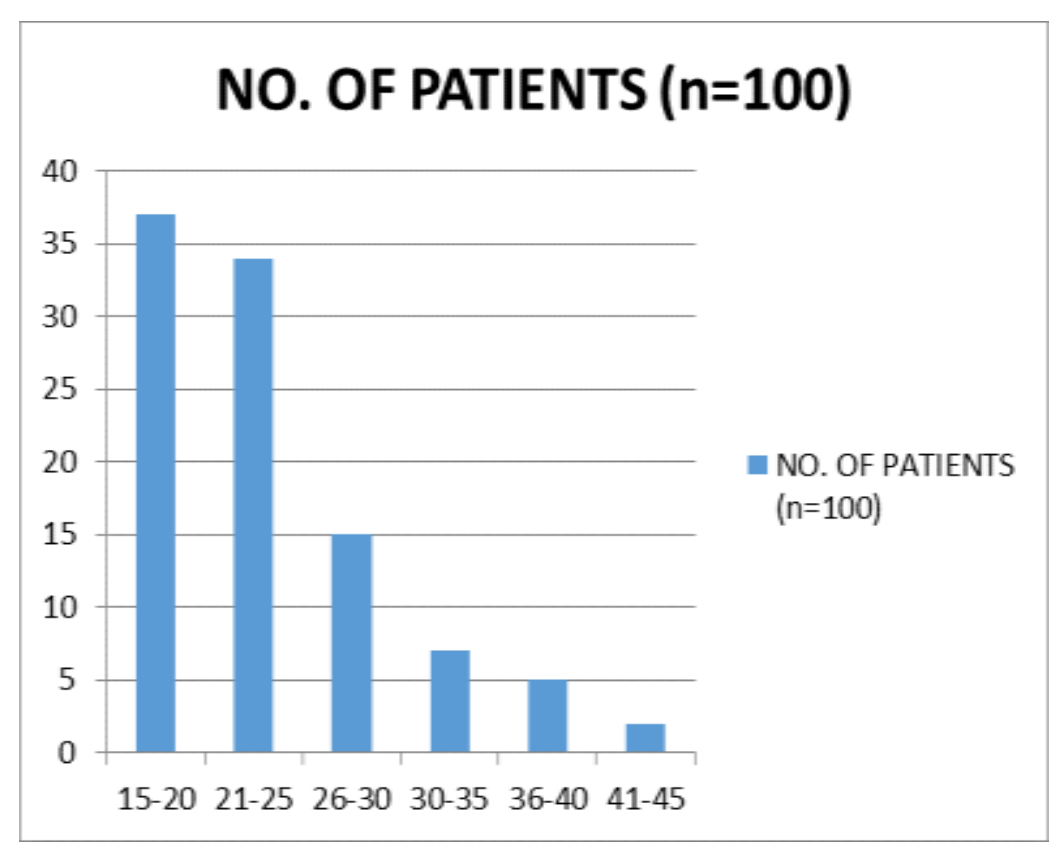

The most common age presenting with mastalgia according to our study is found out to be in age group around 15-20 (37\%) followed by age group between 21-25(34\%). mastalgia is found to be less common among women above 30 years of age. Mean age of presentation in our study is 24 years. The previous series published in Cardiff breast clinic highlights the fact that mastalgia is more a disease of women of reproductive age. The median age of their data was 36 years and their study population which included 212 women of age ranging between 12 and 51 years[14]

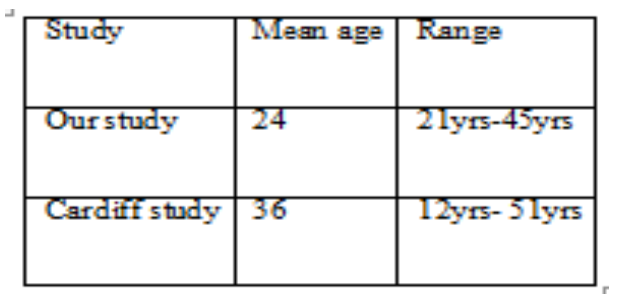




\subsection{Lump and mastalgia}

\begin{tabular}{|l|l|l|}
\hline Lump and Nodularity & Number & Percent \\
\hline PRESENT & 39 & $39 \%$ \\
\hline ABSENT & 61 & $61 \%$ \\
& & \\
\hline
\end{tabular}

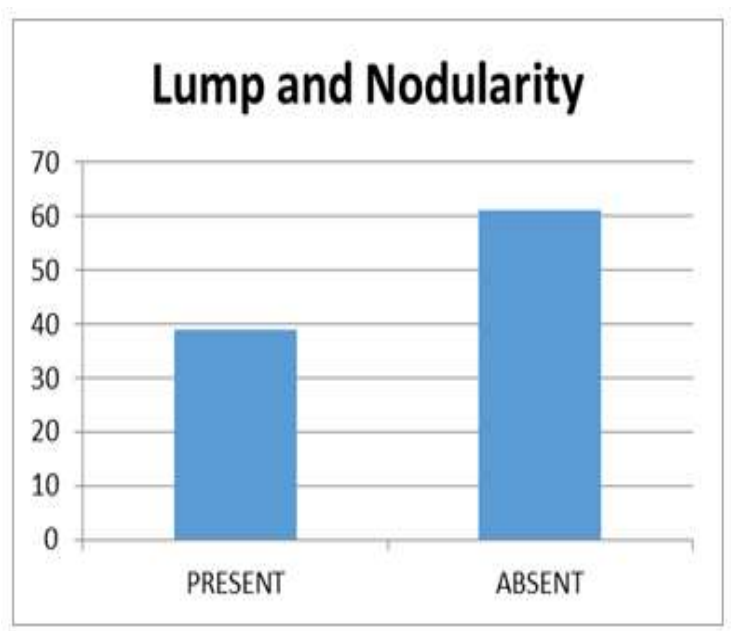

From our observation and clinical examination, 39\% of the patient present with lump or nodularity and rest presented with isolated breast pain alone.

\subsection{Types of mastalgia}

\begin{tabular}{|l|l|l|}
\hline Mastalgia & No. of patient & percentage \\
\hline Cyclic & 80 & $80 \%$ \\
\hline Non cyclic & 20 & $20 \%$ \\
& & \\
\hline
\end{tabular}

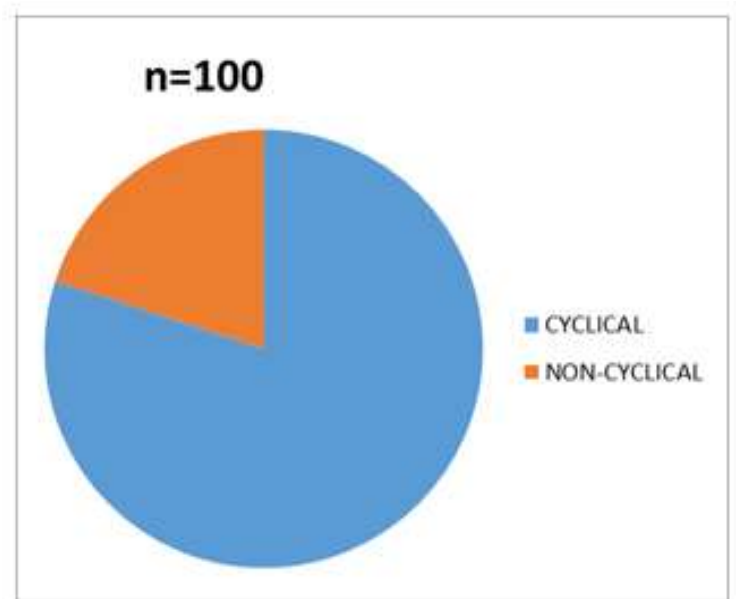

Most of the patient who presented with mastalgia was found to have cyclical type of pain which almost constituted to 80 percent of the women whereas patient with non cyclical mastalgia is around $20 \%$ only.

\subsection{Menstrual cycle in relation to mastalgia}




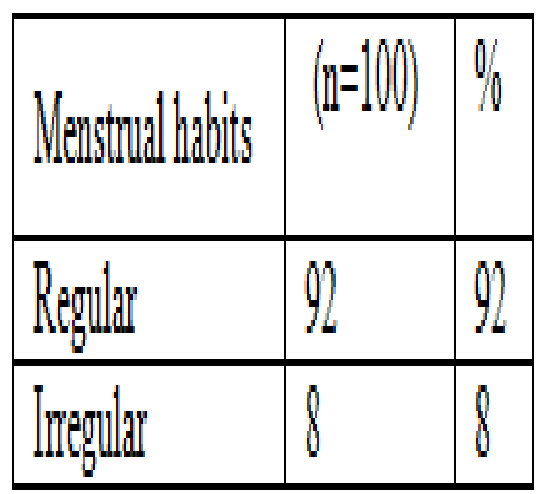

\section{${ }_{8 \%}^{\text {IRREGLAR-MENSTRUALHABITS }}$}

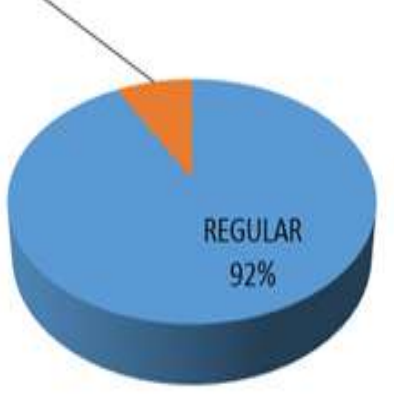

percent of the patient who presented with mastalgia had regular menstrual period when compared to those who presented with irregular period only constituted 8 percent.

\subsection{Dietary habits:}
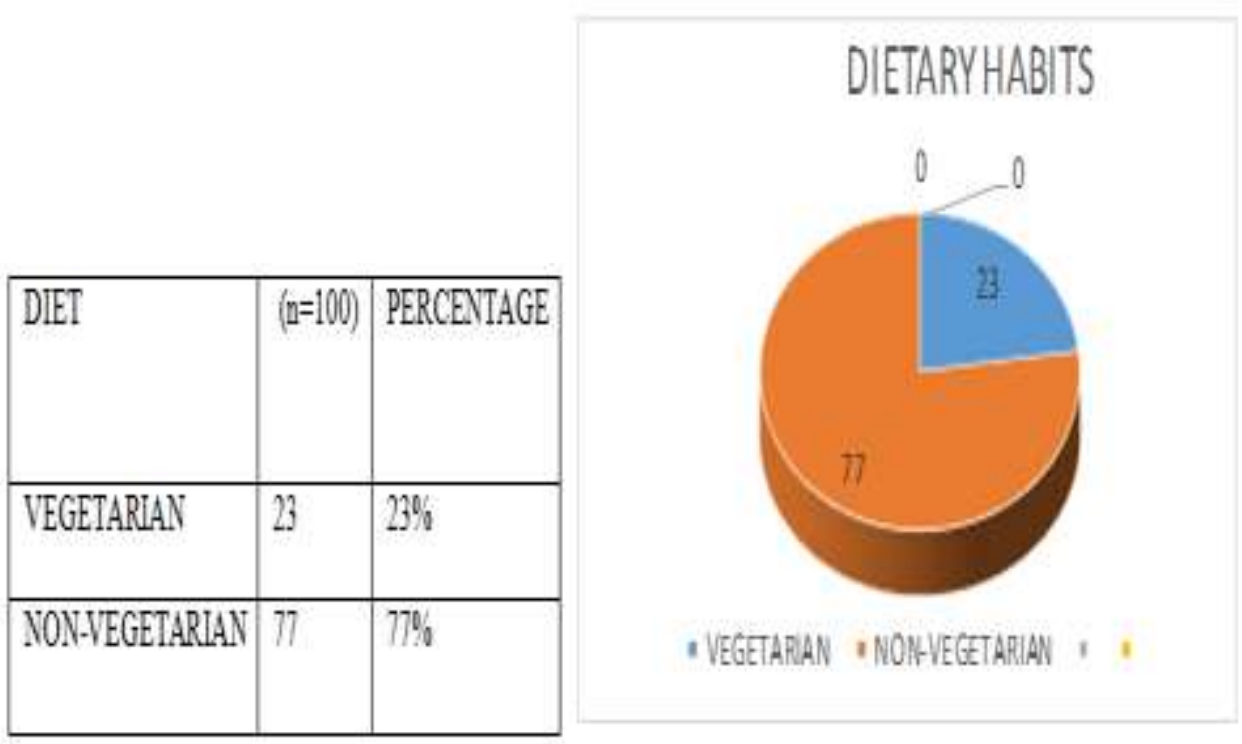

Majority of the patient who presented with mastalgia were those who were on non vegetarian diet almost accounts to 77 percent of the patients whereas vegetarian were only 23percent.Dietary habits may play a role in causing breast pain though there is no strong evidence to rule in or rule out this argument and hence this factor is included in our study. Though most of the women participated in our study were non-vegetarians it is imperative to conduct large population based studies to come to a firm conclusion that non-vegetarian diet is a risk factor for breast pain. In the data published by srivastav et al from AIIMS in which most of the participants were vegetarians it was found that average breast pain scores were higher in non-vegetarian females

\subsection{Breastfeeding and mastalgia}




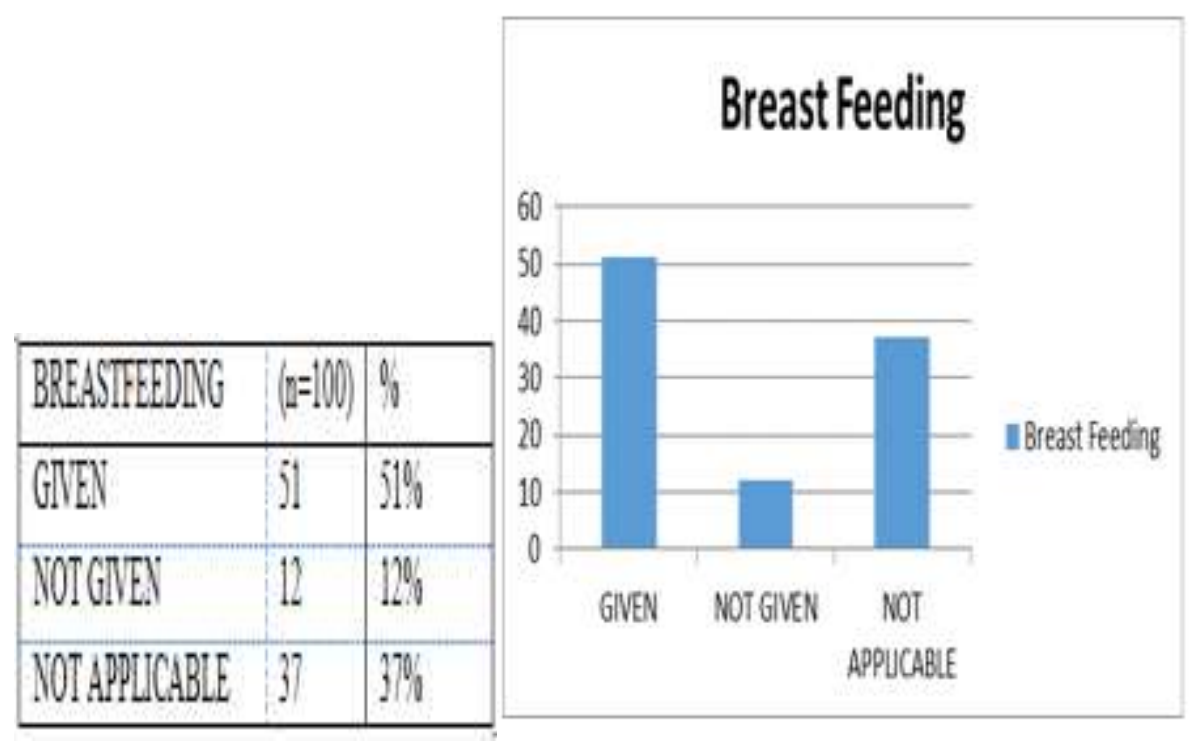

Among those who presented with mastalgia 37 patient were not married and 12 patient had not breastfed their child. However about 51 patients did breastfed their children

\subsection{Body mass index and mastalgia}

\begin{tabular}{|l|l|l|}
\hline BMI & $(\mathrm{n}=100)$ & $\%$ \\
\hline$<20$ & 15 & $15 \%$ \\
\hline $20-24.9$ & 21 & $21 \%$ \\
\hline $25-30$ & 39 & $39 \%$ \\
\hline $30.1-35$ & 23 & $23 \%$ \\
\hline$>35$ & 2 & $2 \%$ \\
\hline
\end{tabular}

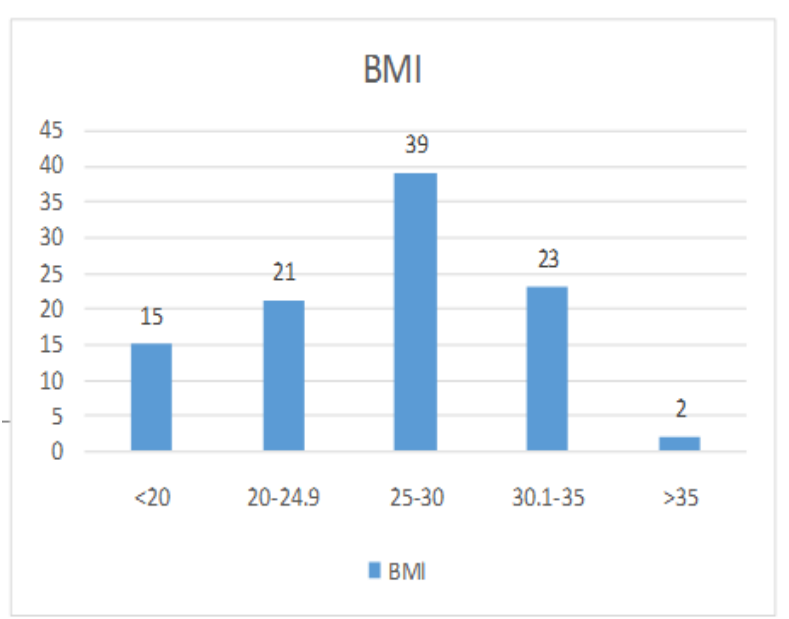

In our study only $36 \%$ of the women had normal BMI and $64 \%$ of them had increased BMI to various degrees but extremely morbid obesity is unusual among women presenting to our hospital which may be due to the fact that this hospital caters to the economically downtrodden people. Obese women are found to have higher pain scores in our study but this need to be confirmed with further large population based studies. Response to treatment is similar to non-obese females and hence it is not a routine schedule in the treatment.

\subsection{Ultrasound findings:}




\begin{tabular}{|l|l|l|}
\hline Treatment & $\mathrm{n}$ & $\begin{array}{l}\text { Avg } \\
\text { Decrease in 6 } \\
\text { months }\end{array}$ \\
\hline Diclofenac gel & 50 & 5.78 \\
\hline Evening primrose oil & 50 & 3.8 \\
\hline
\end{tabular}

\begin{tabular}{|l|l|l|}
\hline USG & $\mathrm{N}=100$ & $\%$ \\
\hline NAD & 67 & $67 \%$ \\
\hline FC & 33 & $33 \%$ \\
\hline
\end{tabular}

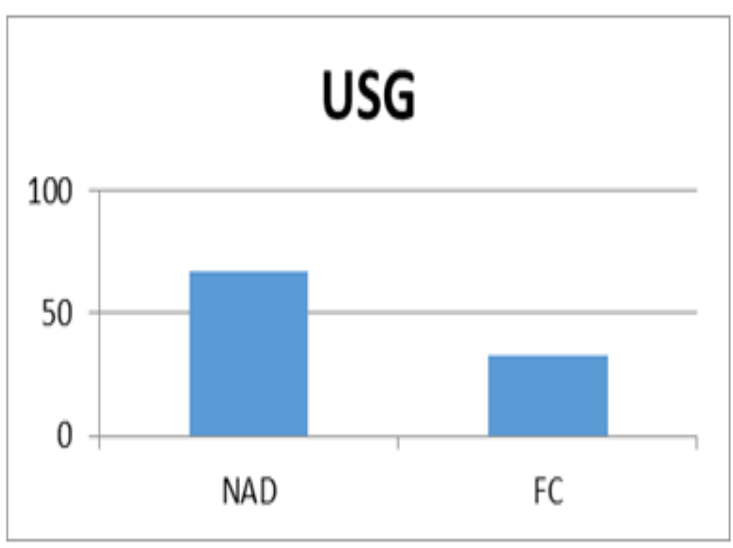

Almost upto 33 percent of the patient had fibrocystic changes when compared to 67 percent of those who underwent ultrasound had no other finding.

\subsection{Treatment of mastalgia}

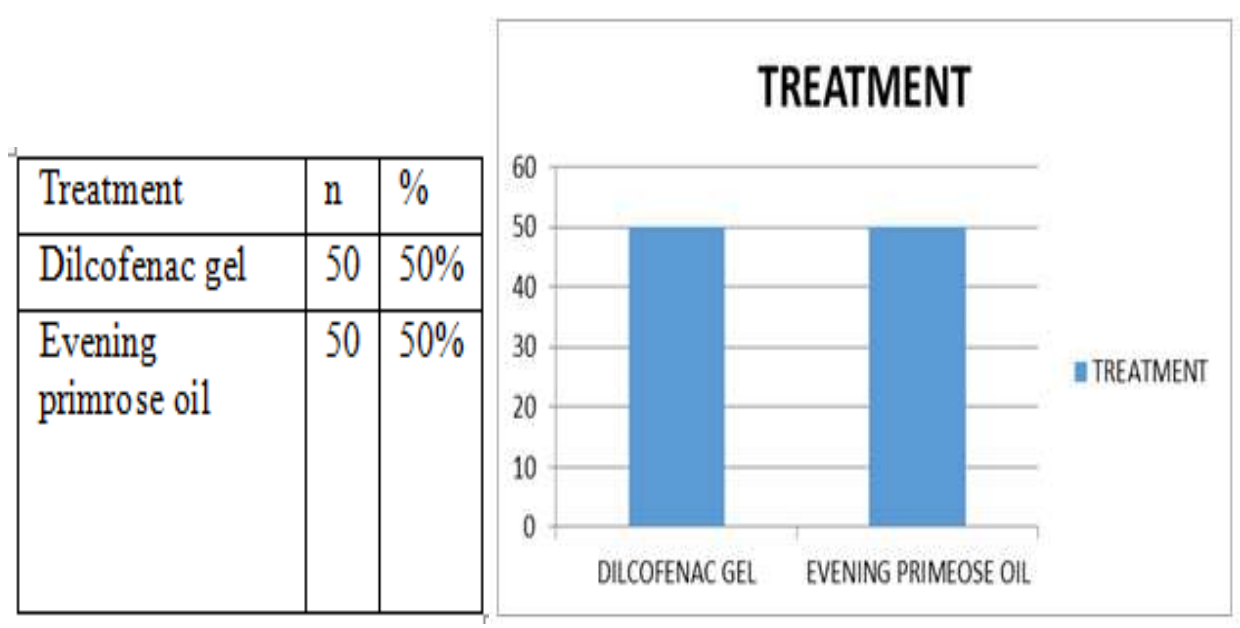

Patient with mastalgia was offered treatment either with topical diclofenac gel and evening primrose oil for a period of three months. Their response was analyzed with help of the visual analogue scoring system which was recorded at the end of first, third and sixth month 


\subsection{Rapid responders}

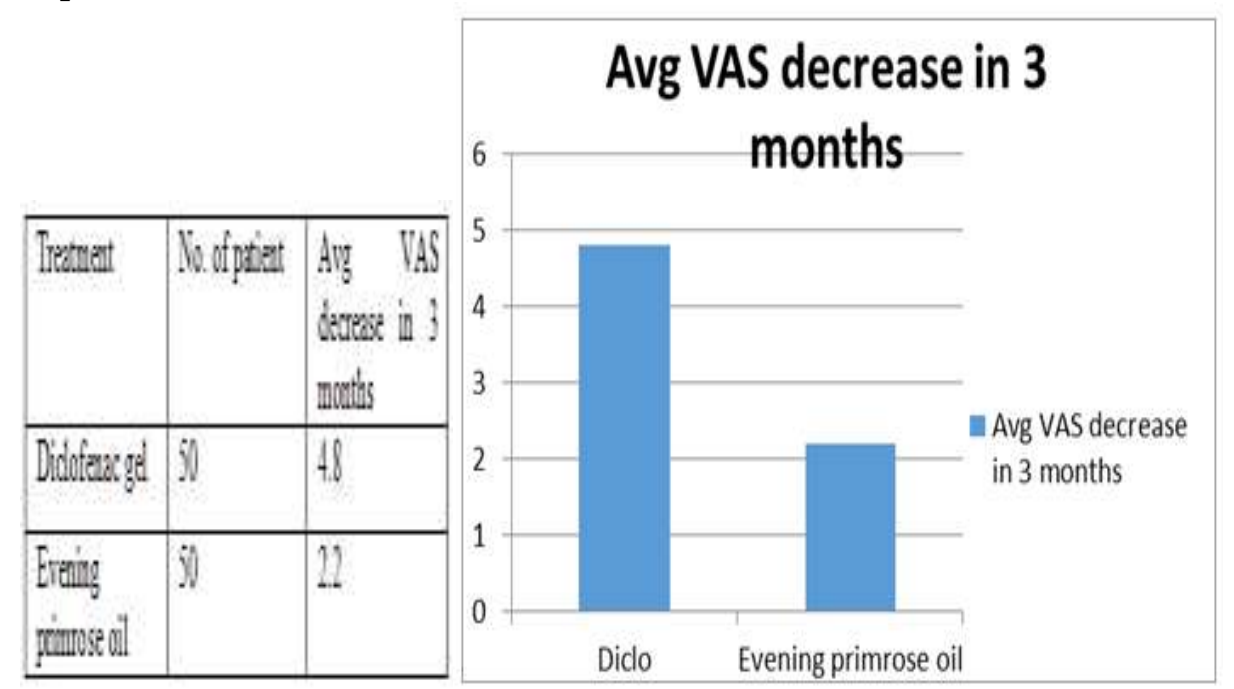

When the response was elicited after a period of 3 months it was found out that average decrease in visual anaologue scale with topical diclofenac gel was 4.8 and with evening primrose oil was found to be 2.2. topical diclofenac gel was found be rapidly acting in management of mastalgia when compared with evening primrose oil.

\subsection{Effectiveness of Topical Diclofenac gel and Evening Primrose oil}

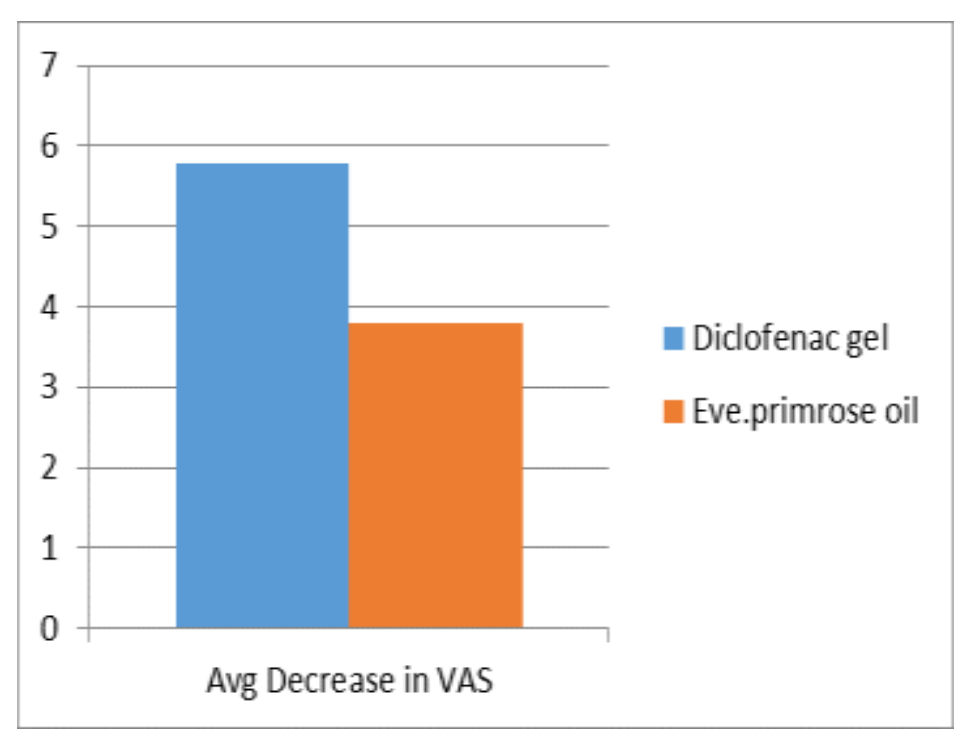

Overall effectiveness of both topical diclefenac gel and evening primrose oil was assessed for a period of 6 months. The average decrease in visual analogue scale in case of topical diclofenac gel was found to be higher (5.78) whereas in evening primrose oil the average decrease was lower (3.8).

12. Overall effectiveness of the treatment:

\begin{tabular}{|l|l|l|l|l|}
\hline \multirow{2}{*}{} & \multicolumn{2}{|l|}{ Diclofenac gel } & \multicolumn{2}{l|}{ Evening primrose oil } \\
\cline { 2 - 5 } & $\mathrm{n}$ & $\%$ & $\mathrm{n}$ & $\%$ \\
\hline Good response & 47 & 94 & 2 & 4 \\
\hline Poor response & 3 & 6 & 48 & 96 \\
\hline
\end{tabular}

\section{Calculation of p value:}

When desired reduction in Visual analogue score is set as minimum of 5 when calculated over a period of 6 months, the following p value was calculated using CHI squared test. However when the effectiveness of both topical diclofenac gel and evening primrose oil, the p value was found to be 0.029 


\begin{tabular}{|l|l|l|l|}
\hline Treatment & Observed value & Expected value & P value \\
\hline Diclofenac gel & 5.78 & 5 & 0.99 \\
\hline Evening primrose oil & 3.8 & 5 & 0.73 \\
\hline
\end{tabular}

\section{Conclusion}

Several findings were listed and the effectiveness of the two drugs were also found as a result of this study.

- Females of reproductive age group are commonly affected with complaint of breast pain.

- Detailed clinical examination and investigations are required when patient presents with lumpiness associated mastalgia .

- Non-cyclical mastalgia is more common in women of south india when compared with cyclical mastalgia but it may need confirmation by further large population based studies.

- Women with mastalgia mostly have regular menstrual cycles.

- Most of these women are non-vegetarians but this may reflect the general population character rather than its association as causality.

- Incidence of breast pain is not affected by breastfeeding.

- Most of the patient who presented with mastalgia were obese.

- Clinical examination is more relevant in terms of ruling out significant pathologies of breast such as cancer rather than as a diagnosing tool. In our study none of these patients showed cancer pointing to the fact that most of the cancerous lumps are painless.

- For better diagnostic yield in assessment of breast lump triple assessment is the best mode of investigation.

- Finally topical diclofenac gel was found to be more effective in management of mastalgia when compared to evening primrose oil,

Since the calculated $\mathrm{p}$ value for both evening primrose oil and topical diclofenac gel is 0.73 and 0.99 which are more than 0.05 , it is observed that both treatments is effective in management of mastalgia.

However when compared with effectiveness between the two drug, the average reduction in Visual Analogue Scale over a period of six months is higher in topical diclofenac gel (5.78) when compared to Evening Primrose oil (3.8). The $\mathrm{p}$ value calculated when both were compared was 0.029 Hence we can come to a conclusion that Topical Diclofenac gel is more effective in treatment of mastalgia when compared to Evening Primrose oil.

\section{References}

[1]. Oksa S, Luukkaala T, Mäenpää J. Toremifene for premenstrual mastalgia: a randomized, placebo-controlled crossover study. BJOG 2006; 113:1-6.

[2]. Tajima C: Luteotropic effects of tamoxifen in infertile women. Fertil Steril 1984; 42:223-7.

[3]. Oksa S, Luukkaala T, Mäenpää J. The antioestrogen toremifene has luteotropic effects in women suffering from premenstrual mastalgia. Acta Endocrinol 2008;

[4]. Oksa S, Parkkola R, Luukkaala T, Mäenpää J. Breast Magnetic Resonance Imaging Findings in Women Treated with Toremifene for Premenstrual Mastalgia. Acta Radiol 2009; 50:984-89.

[5]. Eng-Wong J, Orzano-Birgani J, Chow CK, et al. Effect of raloxifene on mammographic density and breast magnetic resonance imaging in premenopausal women at increased risk for breast cancer. Cancer Epidemiol Biomarkers Prev 2008; 17:1696-1701.

[6]. Odibo AO, Goetzinger KR, Huster KM, Christiansen JK, Odibo L, Tuuli MG. Placental volume and vascular flow assessed by 3D power Doppler and adverse pregnancy outcomes. Placenta 2011; 32:230-4.

[7]. Pomorski M, Zimmer M, Florjanski J, et al. Comparative analysis of placental vasculature and placental volume in normal and IUGR pregnancies with the use of three-dimensional Power Doppler. Arch Gynecol Obstet 2012; 285:331-7.

[8]. Guerriero S, Alcazar JL, Ajossa S, et al. Transvaginal colour Doppler imaging in the detection of ovarian cancer in a large study population. Int J Gynecol Cancer 2010; 20:781-6.

[9]. Zuska J, Crile G Jr., Ayres WW: Fistulas of lactifierous ducts. Am J Surg 81:312, 1951. [PMID: 14819475]

[10]. Kalmantis K, Dimitrakakis C, Koumpis CH, et al. The contribution of three-dimensional power Doppler imaging in the preoperative assessment of breast tumors: a preliminary report. Obstet Gynecol Int 2009; 2009:530579.

[11]. Camiel MR: Mondor's disease in the breast. Am J Obstet Gynecol 152(7 Pt 1):879, 1985. 12. Fisher B, Costantino JP, Wickerham DL, et al. Tamoxifen for the Prevention of Breast Cancer: Current Status of the National Surgical Adjuvant Breast and Bowel Project P-1 Study. J Natl Cancer Inst 2005; 97:1652-62.

[12]. Bilali M, Lagoudianakis EE, Peitsidis $\mathrm{P}$, et al. The role of sonography in the diagnosis of cystic lesions of the breast. Eur $\mathrm{J}$ Gynaecol Oncol 2014; 30:506-8.

[13]. Fisher B, Costantino JP, Wickerham DL, et al. Tamoxifen for the Prevention of Breast Cancer: Current Status of the National Surgical Adjuvant Breast and Bowel Project P-1 Study. J Natl Cancer Inst 2005; 97:1652-62.

[14]. Haagensen CD, et al: Lobular neoplasia (so-called lobular carcinoma in situ) of the breast. Cancer 42:737, 1978. [PMID: 209887] 